\title{
ilmedia
}

Brösel, Gerrit; Keuper, Frank :

Einsatz der Balanced Scorecard im öffentlich-rechtlichen Rundfunk

Zuerst erschienen in:

Controlling \& Management : ZfCM. - Wiesbaden : Gabler, ISSN 16141822 , 48. Jg. (2004), S. 326-334 


\section{Einsatz der Balanced Scorecard im öffentlich-rechtlichen Rundfunk}

Gerrit Brösel/Frank Keuper

\section{Problemstellung}

Die öffentlich-rechtlichen Rundfunkunternehmen sind mehr denn je einem steigenden Handlungsdruck ausgesetz.t. In $\Lambda$ nbetracht der aktuellen wirtschaftlichen Lage und der damit einhergehenden Kostensenkungs- und Sparprogramme über alle Branchen und Unternehmen der Wirtschaft sowie in den meisten privaten Haushalten werden Gebührenerhöhungen der öffentlich-rechtlichen Unternehmen nicht meht widerstandslos akzeptiert. Aktuelle Werbekampagnen privater Rundfunkunternehmen gegen die sog. Rundfunkgebühren der öffentlich-rechtlichen Rundfunkunternehmen im Allgemeinen und die jüngst geplante Gebührenerhöhung im Speziellen bestätigen dies. Rezipienten, aber auch Werbekunden, fordern den im Rundfunkstaatsvertrag kodifizierten wirtschaftlichen und sparsamen Umgang mit den zur Verfügung gestellten Ressourcen. Darüber hinaus führen Branchenentwicklungen, wie digitales Fernsehen oder aber der unaufhaltsame Vormarsch des Internets, zu einer Konvergenz der Anbieterseite und bedingen zunehmend ein wettbewerbsfähiges Handeln. Die unter diesen Aspekten zu berücksichtigende Crossmedialität ist zwar schon in einigen Bereichen der öffentlich-rechtlichen Rundfunkanbieter vorzufinden (z. B. die Verknüpfung von Fernsehprogramm- und
Online-Inhalten), eine generelle Auseinandersetzung mit Visionen und Strategien, welche für den Unternehmenserfolg von entscheidender Bedeutung sind, wird im öffentlich-rechtlichen Rundfunk jedoch noch vielfach vermisst (vgl. Gläser 1999, S. 308 f.). Vor diesem Hintergrund wird nachfolgend ein Konzept einer Balanced Scorecard (BSC) für öffentlichrechtliche Fernsehanbieter vorgestellt, welches einen zentralen $\Lambda$ nsatzpunkt für die Verbesserung der Führung dieser Unternehmen bilden kann.

\section{Hierarchisches Balanced- Scorecard-Konzept}

Hinsichtlich der Organisationsstruktur der verschiedenen Landesrundfunkanstalten hat sich ein nahez.u einheitliches Modell herausgebildet (vgl. u. a. Sieben/ Schwertzel 1997, S.21). Die Leitungsund Aufsichtsorgane umfassen den Intendanten als oberstes Exekutivorgan sowie den Rundfunkrat und den Verwaltungsrat. Die Führungsebene unterhalb des Intendanten ist meist in die "klassischen" Funktionsbereiche „Programmdirektion“, „Produktionsdirektion ", „Technische Direktion" und „Verwaltungsdirektion" unterteilt. Darüber hinaus differenzicren einige Anstalten, wie bspw. der Norddeutsche Rundfunk (NDR), die Programmdirektion in die Bereiche „Fernsehen" und „Hörfunk".
- Die geplante Rundfunkgebütrenerhöhung lasst Zweifel an Wirtschaftlichkeit und Sparsamkeit in Rundfunkanstalten aufkommen. - Ein kaskadiertes Balanced. Scorecard-Konzepl bietet Ansatzpunktê fúr eine Verbesserung der führung von Rundfunkanstalten. - Der Programmauftrag, branchenspezifische Anspruchsgruppen und konvergierende Márkle erfordern eine Anpassung bzw. Subsiitution der ,klassischen" BalancedScorecard-Perspektiven.

- Die sich aus Effektivitäts und Effizienz(an)forderungen ergebenden Ziele werden den gewählten Perspektiven dnspruchsgruppenbzw. dimensionsbezogen zugeordnet.

Diese Struktur liefert einen geeigneten Anhaltspunkt für die Architektur des Balanced-Scorecard-Konzeptes (zu den Grundlagen der BSC vgl. u. a. Kaplan/ Notton 1997 und Horváth \& Partner 2001), welches auch als eine hierarchische Scorecard-Mappe bzw. als eine Corporate Scorecard verstanden werden kann, bei dem die einzelnen Scorecards, wie in Abbildung 1 dargestellt, von oben nach unten kaskadenartig zu entwickeln

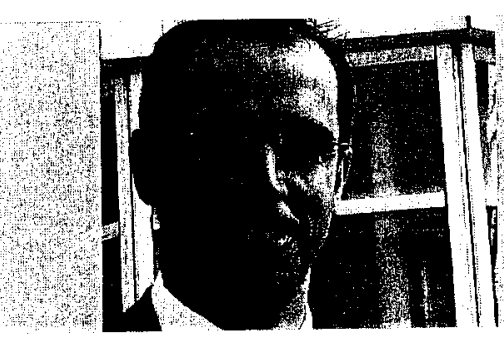

Dr. Gerrit Brösel

Wissensthoftlicher Assistent unid

Habilitand

Technische Universität Ilmendo

Fokültät fur Wirtschiaftswissenschơften
Fachgebiet Rechnutigswesen/

Controlling

Postfacth 100565

98684 Illmeniau
Tel: $03677 / 69-4064$

E-Mail: gerritibroesel@tu-imenaude Internel:

www.konvergenz-management.com 

Effektivität + Effizienz

Effektivität + Effizienz

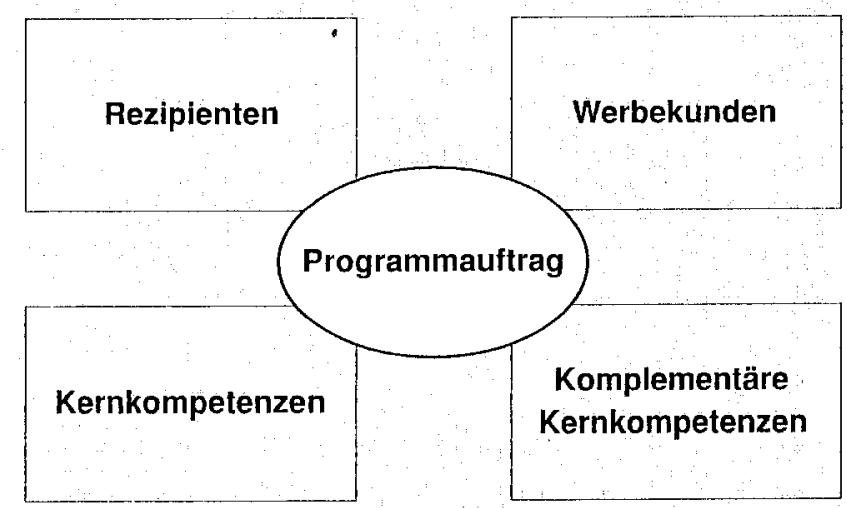

Effektivität + Effizienz

Effektivität + Effizienz

Abbildung 2: Perspektiven der Top-Balanced-Scorecard

anstalt unter Berücksichtigung der individuellen Unternehmensgesamtstrategie, welche eine zwingende Voraussetzung für die Entwicklung der untergeordneten Scorecards darstellt. Die in der Privatwirtschaft als selbstverständlich anzusehende Erarbeitung und Kommunikation der Unternehmensgesamtstrategie und der damit einhergehenden strategischen Ziele wird bei den offentlich-rechtlichen Rundfunkunternehmen noch vielfach vermisst. Dennoch weisen einige Unternehmen erste Scluritte in diese Richtung auf. So positioniert sich das ZDF langfristig nicht mehr als Rundfunk-, sondern als ein Multimedia-Unternehmen, welches den Programmauftrag (vgl. Brösel 2003) bereits in einen Funktionsauftrag überführt hat (vgl. Degenhart 2001, S. 330). Hierbei gilt es, neben den klassischen Aufgaben im Sinne der Programmauftragserfüllung z. B. auch einer Innovationsfunktion nachzukommen (vgl. online ZDF 2003) und damit markt- und ressourcen- bzw. kernkompetenzorientiert der Konvergenz Rechnung zu tragen (Convergence-based View).

Alle individuellen Unternehmensgesamtstrategien der öffentlich-rechtlichen Rundfunkunternehmen haben dabei die bestmögliche Erfüllung der unterschiedlichen Effektivitäts- und Effizienzforderungen zu berücksichtigen und die strate- gischen Erfolgspotenziale, welche Effektivitäts- und Effizienzwirkungen nach sich ziehen, zu entwickeltr. Unter Effektivität wird dabei der Beitrag zur Verbesserung der Wettbewerbsfähigkeit verstanden ("to do the right things"), wohingegen Effizienz das Verhältnis aus erbrachten Leistungen und den dafür eingesetzten Faktormengen charakterisiert. Insofern spiegelt sich die Effizienz im ökonomischen Prinzip wider. Neben der Unternehmensgesamtstrategie erfordert die Konkretisierung der Top-BSC zunächst die Identifizierung der relevanten Perspektiven. Die spezifischen Gegebenheiten und Anforderungen von öffentlich-rechtlichen Rundfunkunternehmen bedingen eine Anpassung beziehungsweise eine Substitution der klassischen Betrachtungsebenen. Unter Berücksichtigung der unterschiedlichen Effektivitäts- und Effizienzforderungen der externen Hauptanspruchsgruppen bilden der Programmauftrag (Anspruchsgruppe: Staat), die Rezipienten und die Werbekunden drei Perspektiven der Top-BSC.

Darüber hinaus sind die eigenen Kernkompetenzen sowie die komplementären Kernkompetenzen der Kooperationspartner - besonders vor dem Hintergrund der Konvergenz auf der Anbieterseite - für den marktlichen Etfolg von Rundfunkanstal- ten von entscheidender Bedeutung. Dies ist darin begründet, dass die Unternehmensgesamtstrategie die aus der Vision abgeleitete globale Wegbeschreibung darstellt und dabei planmäßig festlegt, auf welche Weise strategische Erfolgspotenziale, d. h. distinktive Ressourcen bzw. Kernkompetenzen, aufgebaut bzw. erhalten werden können, um die sich im Umfeld bietenden Chancen unter weitestgehender Abwendung der Risiken auszuschöpfen (vgl. Keuper/Hans 2004, o. S.). Fierbei sind die obersten Unternehmensziele, wie z. B. die Sicherstellung der langfristigen Über lebensfähigkeit, mit Hilfe strategischer Effektivitäts- und Effizienzvorteile verifiziert durch die drei strategischen Erfolgsfaktoren Kosten, Qualität und Zeit - bestmöglich zu erreichen. Insofern werden durch die Unternehmensgesamtstrategie die Märkte und Geschäftsfelder, in denen das Unternehmen tätig scin möchte, definjert und selektiert. Zudem obliegt der Unternehmensgesamtstrategie die Allokation der Ressourcen im Hinblick auf die zu bearbeitenden Märkte und Geschäftsfelder. Die ressourceninduzierte und insbesondere auf emergierende Märkte ausgerichtete Unternehmensgesamtstrategie hat somit durch die Gestaltung ex-ante-orientierter Erfolgspotenziale die Aufgabe, die dynamische und evolutionäre Entwicklung des Unternehmens sicherzustellen. Die Top-BSC muss deshalb um die Kernkompetenzperspektive sowie die Komplementäre Kernkompetenzperspektive ergänzt werden.

Zusammengefasst spannen diese fünf Betrachtungsebenen einen geeigneten Rahmen, mittels dessen sich das relevante Ursache-Wirkungsgefüge auf oberster Unternehmensebene adäquat abbilden lässt (vgl. Abbildung 2). Entgegen dem klassischen Modell von KAPLAN/NORTON beinhaltet die Top-BSC eines öffentlich-rechtlichen Rundfunkunternehmens keine eigenständige Finanzperspektive, was jedoch keine Vernachlässigung einer finanzwirtschaftlichen Betrachtungsweise bedeutet. Die sich aus den Effektivitäts- und Effizienzforderungen ergebenden finanzwirtschaftlichen Ziele werden vielmehr anspruchsgruppenbzw. dimensionsbezogen den bereits 
Programmauftragsperspektive

\begin{tabular}{|c|c|c|c|c|}
\hline \multirow{2}{*}{ Ziele } & \multicolumn{2}{|c|}{ Messgrößen } & \multirow{2}{*}{ Vorgaben } & \multirow{2}{*}{ Strategische Maßnahmen } \\
\hline & Frühindikatoren & Spätindikatoren & & \\
\hline P1: Erhöhung der Vielfalt & $\cdot \ldots$ & $\begin{array}{l}\text { - Programmvolumen je } \\
\text { Sparte }\end{array}$ & $\begin{array}{l}\text { - mind. } \\
20 \% \\
\text { je Sparte }\end{array}$ & $\begin{array}{l}\text { - Erhöhung der Ausstrahlung von } \\
\text { Sendungen im "Bereich Bildung" }\end{array}$ \\
\hline $\begin{array}{l}\text { P2: Verbesserung der Aktualität bei } \\
\text { „Top-Ereignissen“ }\end{array}$ & $\begin{array}{l}\text { - Arzahi/Qualität der } \\
\text { Auslandskorrespon- } \\
\text { denten }\end{array}$ & $\begin{array}{l}\text { - Anzahil der } \\
\text { Rezitationen }\end{array}$ & $+10 \%$ & $\begin{array}{l}\text { - Einstellung/Schulung von } \\
\text { Auslandskorrespondenten }\end{array}$ \\
\hline $\begin{array}{l}\text { P3: Verbesserung der } \\
\text { journalistischen Qualität }\end{array}$ & $\cdot \ldots$ & $\begin{array}{l}\text { - Anzahl der Adolf- } \\
\text { Grimme-Preise }\end{array}$ & $\cdot+5 \%$ & $\begin{array}{l}\text { - Erhöhung der } \\
\text { Mitarbeiterqualifikation }\end{array}$ \\
\hline P4: ... & $\cdot \ldots$ & $\cdot \ldots$ & $\cdot \ldots$ & - $\ldots$ \\
\hline
\end{tabular}

Abbildung 3: Beispieithafie Ausgestallung der Programmauftragsperspektive

dargestellten fünf Perspektiven direkt zugeordnet.

\section{Programmauftragsperspektive der Top-Balanced-Scorecard}

Vor diesem Hintergrund fungiert die Programmauftragsperspektive als die „Basisperspektive“, weil die effektive und effiziente Erfüllung des Programmauftrags die Daseinsberechtigung von öffentlich-rechtlichen Rundfunkunternehmen darstellt (vgl. Brösel 2003). Ausgangspunkt für die Erarbeitung der Ziele, Messgrößen, Zielwerte und strategischen Aktionen bildet die Beantwortung der Frage "Welche Eswartungen des Staates sind durch den Programmauftrag zu erfüllen?". Eine aus staatlicher Sich: zu erfüllende Mindesteffektivität (Sparsamkeitsprinzip), entsprechend dem Grundversorgungsauftrag bei maximaler Effizienz (Wirtschaftlichkeitsprinzip), spannt somit den Rahmen für die zu erarbeitenden Ziele. Dabei ist zu berücksichtigen, dass diese nicht individuell zu konzipieren sind, sondern die gesetzlich zu erfüllenden Anforderungen abbilden. Konkretisiert stellt das „Angebot eines ausgewogenen und vielfältigen Programms in allen Bereichen des Rundfunks" somit eines der zentralen Ziele dar. Abhängig vom bereits bestehenden Programimangebot können die Ziele weiter detailliert werden, so dass die jeweilige Rundfunkanstalt bspw. eine Steigerung der Vielfalt im Programmbereich „Bildung" oder "Information" anstrebt. Messindikatoren zur Steigerung der Vielfalt bilden z. B. die Quote der Wiederholungen oder die Anzahl der unterschiedlichen Zielgruppen, die ein bestimmter Sender anspricht bzw. die mit einem bestimmten Programm erreicht werden (sollen).

Aufgrund des damit einhergehenden Interpretationsspielraumes sowie der aktuellen Diskussion über kontinuierliche Gebührenerhöhungen ist die Konkretisierung dieser Anforderung eines der zentralen Elemente der Top-BSC. So wäre etwa eine Anlehnung an die Ziele aus den Haushaltsgrundsätzen denkbar, bei denen bspw. ein ausgeglichener Haushalt in Form von Einnahmen = Ausgaben oder mindestens eine ausgeglichene Liquidität in Form von Einzahlungen $\geq$ Auszahlungen angestrebt wird. Darïber hinaus bleibt bspw. kritisch zu hinterfragen, inwieweit sich das von den Rundfunkanstalten im Rahmen des Vollprogramms ausgestrahlte (tägliche) Kinderprogramm, trotz Existenz des Kinderkanals (Kika), mit den aus dem Gemeinwirtschaftlichkeitsprinzip abgeleiteten Grundsätzen vereinbaren lässt. In Abbildung 3 sind exemplarisch einige Ziele, deren mögliche Kennzahlen, gegliedert in Früh- und Spätindikatoren, deren Vorgaben sowie strategische Handlungsmaßnahmen aufgeführt.
Rezipientenperspektive der Top-Balanced-Scorecard

Neben der Erfüllung des staatlich vorgegebenen Programmauftrags sind die Rezipienten eine weitere wichtige Anspruchsgruppe im Handlungsfeld von öffentlichrechtlichen Rundfunkunternehmen, weil ihre Anforderungen sich nicht mit denen des Programmauftrags zwingend decken (vgl. Schellhaaß 2000). Eine Abbildung einer Rezipientenperspektive innerhalb der Top-BSC ist somit erforderlich. Im Rahmen der Ansprüche der Rezipienten an ein öffentlich-rechtliches Rundfunkprogramm steht die Beantwortung der Frage, „Wie sollen wir gegenüber unseren Rezipienten auftreten, um deren Erwartungen hinsichtlich Programm und Gebühren zu erfiillen?" im Vordergrund. Mögliche strategische Ziele sind z. B. die Steigerung der Rezipientenzufriedenheit, der Rezipiententreue oder der Gebührenakzeptanz (vgl. Brösel 2003).

Ein bedeutendes Problem stellt auch hier die Festlegung der Messindikatoren sowie deren Überprüfung dar. Neben den klassischen Indikatoren, wie Einschaltquoten und Image eines Unternehmens, lassen sich auch Aussagen mittels weiterer Kennzahlen treffen. So ist bspw. davon auszugehen, dass die Rezipiententreue, ausgedrïckt durch den Anteil an Stammsehern und -hörern, auch Aufschluss über die Gebührenakzeptanz zulässt. Gleicher- 


\begin{tabular}{|c|c|c|c|c|}
\hline \multicolumn{5}{|c|}{ Reziplentenperspektive } \\
\hline \multirow{2}{*}{ Ziele } & \multicolumn{2}{|c|}{ Messgrößen } & \multirow{2}{*}{ Vorgaben } & \multirow{2}{*}{ Strategische Maßnahmen } \\
\hline & Frülinidikat & Spätindikatoren & & \\
\hline $\begin{array}{l}\text { R1: Verbesserung der Akiualität im } \\
\text { Bereich der Unterhaltung }\end{array}$ & $\cdot \cdots$ & $\begin{array}{l}\text { - Quote der wiederholten } \\
\text { Serien }\end{array}$ & $--10 \%$ & $\begin{array}{l}\text { - Erhöhung der Eigenproduktion } \\
\text { von Serien }\end{array}$ \\
\hline $\begin{array}{l}\text { R2: Steigerung des Anteils an } \\
\text { Stammsehem }\end{array}$ & $\cdot \cdots$ & $\begin{array}{l}\text { - Anzahl der Internet- } \\
\text { Portal-Benutzer }\end{array}$ & $\cdot+25 \%$ & $\begin{array}{l}\text { - Ausweitung und verstärkte } \\
\text { Kommunikation des Cross- } \\
\text { media-Angebots }\end{array}$ \\
\hline $\begin{array}{l}\text { R3: Steigerung der } \\
\text { Rezipientenzufriedenlieit }\end{array}$ & $\begin{array}{l}\text { Zufriedenheits- } \\
\text { umfragen }\end{array}$ & $\bullet$ & $\cdot+8 \%$ & $\begin{array}{l}\text { - Ausweitung der Integration der } \\
\text { Rezipienten bei der Programm- } \\
\text { gestallung }\end{array}$ \\
\hline R4: ... & $\cdot \ldots$ & - $\ldots$ & - $\ldots$ & $\cdot \ldots$ \\
\hline
\end{tabular}

Abbildung 4: Beispielhafte Ausgestaltung der Rezipientenperspektive

maßen spiegelt sich die Reripientenzufriedenheit nicht nur in der absolut erzielten Einschaltquote, sondern auch in der relativen Attraktivität einer Sendung, also im erzielten Marktanteil an einer bestimmten Zielgruppe, wider. In Rahmen der Rezipiententreue ist weiterführend zu hinterfragen, inwieweit sich diese auf den Sender allgemein oder aber auf bestimmte Serien oder Sparten, wie z. B. den Freitagskrimi im ZDF, bezieht. Die Abbildung 4 stellt einige mögliche Ziele, Kennzahlen, Vorgaben und Maßnahmen dar, die in einer Top-BSC verankert werden könnten.

\section{Werbekundenperspektive der Top-Balanced-Scorecard}

Als eine weitere Anspruchsgruppe der öffentlich-rechtlichen Rundfunkunternehmen sind die Werbekunden zu berücksichtigen. Die Beantwortung der Frage, "Wie bzw. mit weichem Programm sollen wir gegenüber unseren Werbekunden auftreten, um auch weiterhin Werbeeinnahmen zu erzielen?" bildet den Ausgangspunkt für die Erarbeitung der Ziele und strategischen Aktionen dieser Werbekundenperspektive. Ein Ziel dieser Betrachtungsebene ist bspw. die Steigerung des Anteils der Werbecinnalumen an den Gesamteinnahmen. Dies lässt sich u.a. durch eine Ausweitung der Werbeminuten unter Beachtung der Regelungen des RStV oder durch erhöhte Werbegrundpreise erzielen.

In diesem Zusammenhang muss die Werbeerlös-Reichweiten-Spirale berücksichtigt werden, welche in der Regel mit einer Ausweitung der Anzahl der erreichten Personen initiiert wird. Daraus resultierende sinkende Tausender-Kontaktpreise führen zunächst zu einem steigenden Anzeigenvolumen (Mengeneffekt).

\begin{tabular}{|c|c|c|c|c|}
\hline \multicolumn{5}{|c|}{ Werbekundenperspektive } \\
\hline Ziele & Frühindikatoren & Spảtindikatoren & Vorgaben & Strategische Maßnahmen \\
\hline W1: Steigerung der Werbeeinnahmen & $\begin{array}{l}\text { - Anzahl der Anfragen } \\
\text { nach innovativen } \\
\text { Werbekonzepten }\end{array}$ & $\begin{array}{l}\text { - Einnahmen durch } \\
\text { Werbung, Werbe- } \\
\text { grundpreise }\end{array}$ & $++10 \%$ & $\begin{array}{l}\text { - Akquisition zielgruppen- } \\
\text { spezifischer Rezipienten }\end{array}$ \\
\hline $\begin{array}{l}\text { W2: Verbesserung der Werbekunden- } \\
\text { zufriedenheit }\end{array}$ & $\begin{array}{l}\text { - Prognosen über zu } \\
\text { erwartende Reichweilen }\end{array}$ & $\cdot \ldots$ & $\begin{array}{l}\text { - mind. } \\
+5 \%\end{array}$ & $\begin{array}{l}\text { - Regelmäßig zuverlässige und } \\
\text { aussagefähige Prognosen } \\
\text { durchführen }\end{array}$ \\
\hline $\begin{array}{l}\text { W3: Verbesserung des Images durch } \\
\text { ausgewählle Werbung }\end{array}$ & - $\ldots$ & $\begin{array}{l}\text { - Anzahl von "Image- } \\
\text { trägern" der Werbe- } \\
\text { wirtschaft }\end{array}$ & $\cdot+5 \%$ & $\begin{array}{l}\text { - Akquisition von gesellschaftlich } \\
\text { angesehenen Werbekunden, } \\
\text { wie bspw. Kirche, UNICEF etc. }\end{array}$ \\
\hline W4:... & - $\ldots$ & - ... & $\cdot \ldots$ & * $\cdots$ \\
\hline
\end{tabular}

Abbildung 5: Beispielhaffe Ausgestalfung der Werbekundenperspektive 
Kernkompetenzperspektive

\begin{tabular}{|c|c|c|c|c|c|}
\hline \multicolumn{6}{|c|}{ Kernkompetenzperspektive } \\
\hline \multicolumn{2}{|r|}{ Ziele } & Frühindikatoren & Spätindikatoren & Vorgabe & Strategische Maßnahmen \\
\hline & $\begin{array}{l}\text { Verstärkung des regionalen } \\
\text { Bezugs }\end{array}$ & $\cdot \cdots$ & $\begin{array}{l}\text { - Anteil „regionaler" } \\
\text { Programmhersteilung }\end{array}$ & $\cdot+10 \%$ & $\begin{array}{l}\text { - Erhöhung der Mitarbeiterzahl und der } \\
\text { Eigenproduktionen für regionales } \\
\text { Programrn }\end{array}$ \\
\hline K2: & Verbesserung der Unabhängigkeit & - $\ldots$ & $\begin{array}{l}\text { - Anteil der Eigen- } \\
\text { produktionen }\end{array}$ & $\cdot+5 \%$ & $\begin{array}{l}\text { - Zukauf neuer } \\
\text { Produktionskapazităten }\end{array}$ \\
\hline & $\begin{array}{l}\text { Verbesserung der Präsentation } \\
\text { von Nachichten }\end{array}$ & $\begin{array}{l}\text { Weiterbildungsmaß- } \\
\text { nahmen der } \\
\text { Moderatoren }\end{array}$ & - ... & $.8 \%$ & $\begin{array}{l}\text { - Ausweitung der Weiterbildungs- } \\
\text { maßnahmen von Moderatoren }\end{array}$ \\
\hline K4: & $\ldots$ & - $\ldots$ & - $\ldots$ & - $\ldots$ & - $\ldots$ \\
\hline
\end{tabular}

Abbildung 6: Beispielhaffe Ausgestalıung der Kernkompetenzperspektive

Mittelfristig können sich jedoch die Werbgrundpreise erhöhen lassen (Preiseffekt), weil die gesunkenen TausenderKontaktpreise die Spielräume für eine Preiserhöhung schaffen (vgl. Heinrich 2001, S. 241; Köcher 2002, S. 43). Darüber hinaus gibt der Anteil der Werbeerlöse einer Rundfunkanstalt an den Gesamtbrutto-Werbeerlösen Aufschluss über die relative Attraktivität der Anstalt gegenüber der Konkurrenz. Abbildung 5 zeigt beispielhaft Ziele, Messgrößen, deren Vorgaben und Maßnahmen der Werbekundenperspektive auf.

\section{Kompetenzperspektive der Top-Balanced-Scorecard}

Neben den Perspektiven aus Sicht der externen Anspruchsgruppen ist in der TopBSC die Kernkompetenzperspektive zu verankern, der alle intrabetrieblich relevanten Faktoren subsumiert werden. Bei Kernkompetenzen handelt es sich um unternehmensindividuell konfigurierte distinktive Ressourcen (vgl. Prahalad/ Hamel 1990, S.83 f.; Börner 2000, S. 79), welche die Basis für strategische Wettbewerbsvorteile bilden. Distinktive Ressourcen umfassen dabei sämtliche Quellen. eines Unternehmens, die für die Effektivität und Effizienz des Unternehmens verantwortlich sind und gleichzeitig die Eigenschaft der Wertgenerierung, der Einzigartigkeit und der Nicht-Imiticrbarkeit innehaben (vgl. Bürki 1996, S.202 ff.). Eine Kernkompetenz ist somit eine Konbination von sich ergänzenden Fähigkeiten und Wissensgrundlagen, die in der Organisation eingebettet sind und dazu führen, dass einer oder mehrere kritische Unternehmensprozesse auf höchstem Niveau beherrscht werden (vgl. Abplanalp/Lombriser 2000, S. 52).

Wesentliche Faktoren der Kernkompetenzen stellen dabei in der Regel die individuelle Strategie (immaterielle Kompetenz) oder aber die eingesetzten Technologien und die Organisation (sowohl der eigentliche Aufbau als auch die intra- und interbetrieblichen Abläufe) dar. Zusätzlich fungieren auch die Mitarbeiter hinsichtlich ihrer Qualifikationen, aber auch bezüglich ihres zwischenmenschlichen Ungangs, ausgedrïckt durch die Unternehmenskultur, als wesentliche Quellen der Kernkompetenzen. Unter Berücksichtigung des Zusammenspiels dieser Faktoren ist die Erstellung von programmauftragskonformen Inhalten (also Nachrichten, Reportagen, Serien, Kurzmeldungen, Verkehrsinformationen etc.) sowie deren Bündelung und Ausstrahlung als Kerngeschäft von öffentlichrechtlichen Rundfunkunternehmen anzusehen. Auf dem Kerngeschäft basierende Kernkompetenzen besitzt ein öffentlichrechtliches Rundfunkunternehmen dann, wenn es den Anspruchsgruppen einen besonderen Nutzen stiftet bzw. sich gegenüber den Wettbewerbern abhebt.

Verglichen mit dem ZDF zeichnet sich der NDR z. B. durch seine regionale Verankerung aus. Durch zahlreiche lokale Berichterstattungen wird für eine bestimmte Zielgruppe - nämlich die Rezipienten eben dieser Region - zusätzlicher Nutzen geschaffen. Die Strategie der - in weiten Teilen - regional ausgerichteten Progranimgestaltung stellt eine Kernkompetenz des NDR dar (Portfolio-Strategie). Gleichermaßen besitzt der NDR, u. a. mit seinen Itamburger Filmstudios, eigene redaktionelle, aber auch technische Produktionskapazitäten, die in gewissem Maße eine Unabhängigkeit gegenüber Fremdanbietern von Programm-Produktionen ermöglichen. Die Herstellung von Eigenproduktionen ist somit ebenfalls dem Kerngeschäft des NDR zuzuordnen. Darüber himaus verfügen $z$. B. der NDR und der WDR über eine eigenständige Hörfunk-Sparte als weiteren Vertriebskanal. Gegenüber den privaten Anbietern und dem ZDF werden folglich die Inhalte nicht nur über das Fernsehen und das Internet, sondern zusätzlich auch noch über das Radio vertrieben.

Innerhalb der Kernkompetenzperspektive lassen sich somit vielfältigste Messwerte und Kennzahlen definieren. Strategisches Ziel des NDR könnte u.a. ein noch stärkerer regionaler Bezug innerhalb 


\begin{tabular}{|c|c|c|c|c|}
\hline \multicolumn{5}{|c|}{ Perspektive der komplementäre Kernkompetenzen } \\
\hline \multirow{2}{*}{ Ziele } & \multicolumn{2}{|c|}{ Messgrößen } & \multirow{2}{*}{ Vorgaben } & \multirow{2}{*}{ Strategische Maßnahmen } \\
\hline & 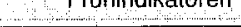 & Spatinc & & \\
\hline $\begin{array}{l}\text { KK1: Verbesserung der Zusammen- } \\
\text { arbeit mit technischen } \\
\text { Kooperationspartnern }\end{array}$ & $\cdot \ldots$ & - Anzahl der SLA & $\cdot+10 \%$ & $\begin{array}{l}\text { - Abschließen von langfristigen } \\
\text { Rahmenverträgen; Fixieren der } \\
\text { Service Levels }\end{array}$ \\
\hline $\begin{array}{l}\text { KK2: Steigerung der Markenbildung } \\
\text { von Personen }\end{array}$ & - Imageentwicklung & $\cdot \ldots$ & $\cdot+5 \%$ & $\begin{array}{l}\text { - Gezielte Marketingmaßnahmen } \\
\text { zum Ausbau der Person als Marke }\end{array}$ \\
\hline $\begin{array}{l}\text { KK3: Senkung von Gemeinschafts- } \\
\text { produktionskosten }\end{array}$ & - $\ldots$ & $\begin{array}{l}\text { - Benchmark der Fremd- } \\
\text { kosten mit anderen } \\
\text { öffentlich-rechtlichen } \\
\text { Rundfunkunternehmen }\end{array}$ & $\begin{array}{l}\text { - unter den } \\
\text { drei } \\
\text { Besten }\end{array}$ & $\begin{array}{l}\text { - Identifizierung der Kooperations- } \\
\text { Kostentreiber, Neuverhandlung } \\
\text { der bestelendenden Verträge oder neuer } \\
\text { Kooperationen }\end{array}$ \\
\hline KK4: ... & - $\ldots$ & - ... & - $\ldots$ & - $\cdots$ \\
\hline
\end{tabular}

Abbildung 7: Beispielhafte Ausgestaltung der Perspektive der komplementären Kernkompetenzperspektive

der Programmgestaltung scin. Dies wiederum erfordet aber eine Erhöhung der Anzahl der Redakteure und Mitarbeiter, die sich mit der regionalen Programmherstellung beschäftigen. Bezogen auf die Ebene der Mitarbeiter bieten sich für eine Rundfunkanstalt vielfältige Möglichkeiten zum Erhalt bzw. zum Ausbau von Kernkompetenzen. Umfasst bspw. die ヘusbildung als Moderator in einem Rundfunkunternehmen spezifische und erstklassige Weiterbildungsmaßnahmen, wie Rhetorikkurse oder aber sogar Auslandseinsätze, so kann dies nicht nur zu einer verbesserten Moderatorenleistung und somit zu einer besseren Programmdarstellung beitragen, sondern gleichzeitig auch das Image des Unternehmens als attraktiver Arbeitgeber fördern.

Vor dem Hintergrund der aktuellen wirtschaftlichen Lage sowie der mangelnden Akzeptanz für steigende Rundfunkgebühren sehen sich auch öffentlich-rechtliche Rundfunkunternehmen gezwungen, ihre Kosten zu senken. Gerade bei Filmproduktionen variieren die Produktionskosten einer Sendung pro Minute enorm. Ziel eines öffentlichrechtlichen Rundfunkuntcrnchmens könnte z. B. die Reduzierung der Kosten je Sendeminute von Filmproduktionen um 10\% sein. In Abbildung 6 sind exemplarisch mögliche Ziele, Messgrößen, Vorgaben und Handlungsmaßnahmen

der Kernkompetenzperspektive aufgefülırt.

\section{Perspektive der komplementären Kernkompetenzen der Top-Balanced-Scorecard}

Im Rahmen der komplementären Kernkompetenzen gilt es, bestehende unternehmenseigene Kernkompetenzlücken durch das Eingehen von strategischen Kooperationen mit anderen Unternehmen oder Personen zu schließen (vgl. Keuper/ Hans 2003, S. 85 f.). Die hierdurch zum Ausdruck kommende angebotsseitige Konvergenz unterstützt die effolgreiche Vetfolgung der Crossmedia-Strategie. Im Hinblick auf die Perspektive der komplementären Kernkompetenzen bedeutet dies die Beantwortung der Frage, „Mit welchen Unternehmen und Personen sollen Kooperationen aufgebaut bzw. aufrecht erhalten werden, um die Kernkompetenzlücken zu schließen und letztlich Erfolg zu erzielen".

Ausgangspunkt der Betrachtung ist zunächst das Identifizieren der Kernkompetenzlücken von öffentlich-rechtlichen Rundfunkunternehmen. Einen wesentlichen Bereich der komplementären Kernkompetenzen stellen hierbei die „Neuen Medien" dar. Noch vor einigen Jahren beschränkte sich die Erfüllung des Programmauftrags auf die Bereiche „Fernsehen “ und „Hörfunk", so dass davon aus- zugehen ist, dass viele der öffentlichrechtlichen Rundfunkunternehmen im Bereich des Internets zwar Kompetenz, jedoch noch keine Kernkompetenz aufbauen konnten. Daher bieten sich strategische Kooperationen mit Unternehmen an, deren Kernkompetenzen beispielsweise im Marketing oder aber in der technischen Ausstattung und Abwicklung des OnlineAuftritts liegen. Dies wird auch durch die Kooperation zwischen dem ZDF und dem Untemehmen Microsoft, welches als Service Provider für den Online-Auftritt des ZDF fungiert, bestätigt. Konkretisiert für die Top-BSC ist bspw. ein ZuverlässigkeitsIndex anwendbar, der die Zuverlässigkeit hinsichtlich Leistung bzw. die Fehlerquote des Internetseitenaufrufs beschreibt und in einem sog. "Service Level Agreement" (SLA) verankert wird. "Service Level Agreements", welche aktuell besonders im EDV-Bereich Anwendung finden, in der Regel aber für jede Kooperation abgeschlossen werden können, determinieren das gegenseitige Anspruchsniveau des Leistungsverhältnisses und beeinflussen somit die Qualität der Kooperation.

Darüber hinaus können auch sog. Imageträger; wie z. B. Thomas Gottschalk oder Sabine Christiansen, von Rundfunkunternehmen zu den Kooperationspartnern gezählt werden. Als Sympathieträger beeinflussen sie das Verhalten der Rezipienten und Werbekunden und führen 


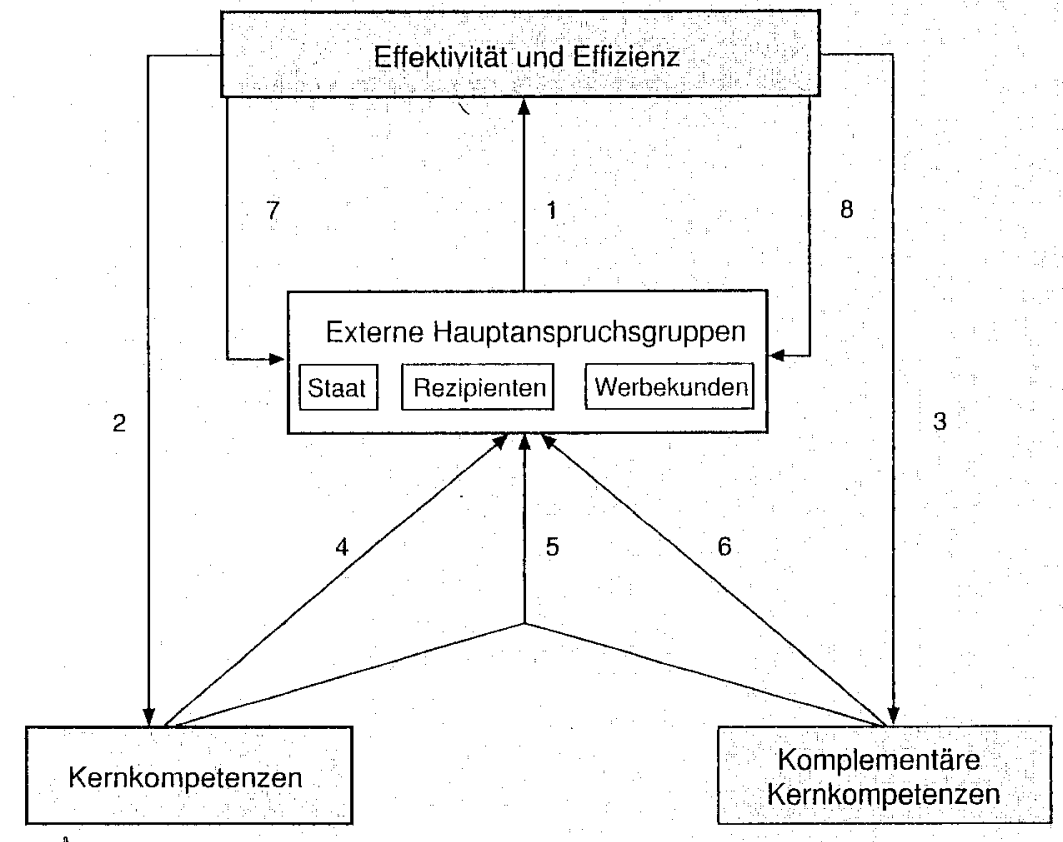

Abbildung 8: Ursache-Wirkungsgefüge zwischen den Perspektiven der Top-BSC

u. U. zu höheren Reichweiten, steigenden Werbeeinnahmen oder generell zur Imageverbesserung des Rundfunkunternehmens. Bei der Verankerung dieser Imageträger in einer Top-BSC ergibt sich jedoch die Schwierigkeit der Quantifizierung des Nutzens, den diese Kooperationspartner stiften. Mögliche Indikatoren zur Messung der Beliebtheit dieser Sympathieträger sind neben den von ihnen erzielten Einschaltquoten auch die Anzahl der „Fanpost", das Interesse der Werbebranche sowie die Abwerbungsversuche von Konkurrenzuntemehmen. Mögliche Ziele, Messgrößen, Vorgaben und Handlungs maßnahmen der Perspektive der komplementären Kernkompetenzen sind in $\mathrm{Ab}$ bildung 7 exemplarisch dargestellt.

\section{Mögliche Ursache-Wirkungs ketten der Top-Balanced- Scorecard}

Nachdem sämtliche Ziele, Messwerte und strategischen Aktionen für alle Perspektiven durch die Führungsebene der Rundfunkanstalt identifiziert wurden, gilt es, die spezifischen Ursache-Wirkungsbezie- hungen herzustellen und somit die Perspektiven miteinander zu verknüpfen. Abbildung 8 verdeutlicht - stark aggregiert die Dependenzen und Interdependenzen z.wischen den Perspektiven und den Effizienz- und Effektivitärsaspekten. Die nachfolgenden Beispiele sollen einen Einblick in die möglichen Ursache-Wirkungsketten geben und dienen als Ansatz für die Konzeption einer konkreten Top-BSC. Dabei sind insbesondere die individuellen Strukturen, Kernkompetenzen und verfolgten Strategien zu berücksichtigen. Weiterführend ist dann eine Verzahnung mit den einzelnen (separat zu konzipierenden) Direktions-Scorecards vorzunehmen.

Ausgehend von den Erwartungen des Staates sowie der Rezipienten und Werbekunden ergeben sich spezifische Effektivitäts- und Effizienzforderungen an öffentlich-rechtliche Rundfunkunternelımen (Pfeil 1), welche es im Rahmen der Leistungserstellung mittels der Kernkompetenzen und komplementären Kernkompetenzen zu erfüllen gilt (Pfeile 2 und 3). Unter Berücksichtigung der Effektivitäts- und Effizienzaspekte werden auf Basis der Kernkompetenzen sowie mittels einge- gangener Kooperationen Produkte und Leistungen hergestellt, die direkt auf die Rezipienten und Werbekunden einwirken bzw. die Anforderungen des Staates erfüllen sollen (Pfeile 4, 5 und 6). In $\Lambda$ bhängigkeit von den bei den externen Hauptanspruchsgruppen subjektiv empfundenen Wirkungen erfolgt ein individueller $\mathrm{Ab}$ gleich zwischen den gestellten Erwartungen und dem erzielten Erfüllungsgrad, der gegebenenfalls Veränderungen der Effektivitäts und Effizienzforderungen nach sich zieht (Pfeile 1,7 und 8) und welche an die Kernkompetenzen und komplementären Kernkompetenzen weiterzugeben sind (Pfeile 2 und 3).

Konkretisiert kann so bspw. der Zusammenhang zwischen Marktforschungsaktivitäten und einer verbesserten Programmqualität dargestellt werden. Werbekunden schalten ihre Werbung nur, wenn die mit der Werbung forcierte Zielgruppe mit der Zielgruppe der Rezipienten übereinstimmt. Dies impliziert für öffentlich-rechtliche Rundfunkuntemehmen den Besitz von (Kern-)Kompetenz im Bereich der Marktforschung. Verbessert ein öffentlich-rechtliches Rundfunkunternehmen seine Marktforschungsaktivitäten hinsichtlich der Zielgruppenidentifizierung und deren Zuordnung zum forcierten Programm, so lässt sich dieses individueller auf die Rezipientenwiinsche anpassen. Eine damit einhergehende erhöhte Rezipientenreichweite führt sowohl zu einer besseren Befriedigung der Erwartungen der Werbekunden als auch zu sinkenden Tausender-Kontaktpreisen. Aus Sicht der öffentlichrechtlichen Rundfunkunternehmen lassen sich mittelfristig aufgrund des gesteigerten Interesses der werbetreibenden Wirtschaft höhere Werbegrundpreise crzielen. Die erhöhten Werbeeinahmen wiederum bieten die Möglichkeit, finanzielle Mittel in neue respektive bessere Produktionen zu investieren, was aus Sicht der Rezipienten mit einer Verbesserung der Programmqualität einhergeht (vgl. WerbeerlösReiclıweiten-Spirale).

Eine weitere Ursache-Wirkungskette lässt sich zwischen dem Aufbau von Kompetenz im Online-Bereich und einer Erhöhung der Anzahl der Rezipienten 
beschreiben. Umfassende und nicht nur sendungsbezogene Internetangebote werden heute auch von öffentlich-rechtlichen. Unternehmen erwartet. Neben der Erfüllung dieser Erwartung lässt sich aber auch zielgruppenorientiert das Interesse potenzieller Rezipienten auf bestimmte Programme oder Sendungen lenken, die somit direkt als neue Kunden gewonnen werden können. Gleichermaßen führt ein professioneller und umfassender Internetauftritt auch zu einer Verbesserung des Images und der Bekanntheit einer Rundfunkanstalt, was wiederum positiv auf die Rezipientengewimnung und -bindung wirkt.

\section{Zusammenfassung und Ausblick}

Öffentlich-rechtliche Rundfunkanbieter sehen sich zunelumend einem steigenden Handlungsdruck ausgesetzt. Die wirtschaftliche und sparsame Erfüllung des Programmauftrags bei gleichzeitiger Befriedigung der Interessen der Rezipienten und der Werbewirtschaft scheint zunehmend unerfüllbar. Darüber hinaus weisen die neuerlichen Überlegungen, die monatlichen "Rundfunkgebühren " zum Jahre 2005 um 1,09 Euro auf 17,24 Euro anzuheben, auf Verschwendungen im Umgang mit den finanziellen Ressourcen und folglich auf Schwächen in der Unternehmensführung und-steuerung von öffentlich-rechtlichen Rundfunkanstalten hin. Zunehmend werden Forderungen nach einem umfassenden Controlling laut, mittels dessen sich die Planung, Kontrolle und Informationsversorgung im Hinblick auf die Unternehmenszielsetzung erfolgreich gestalten, vor allem aber messen lässt. Da bei näherer Betrachtung derzeitig verwendcter Instrumente zu erkennen ist, dass bis heute nur einfache Systeme zur Unternehmenssteuerung eingesetzt werden, die eine einseitige Finanz- und Vergangenheitsorientierung und eine mangelnde Strategieausrichtung aufweisen und dem tatsächlichen Spannungsverhältnis von Programmauftrag, Werbewirtschaft und Rezipienten nicht gerecht werden, ist die Entwicklung integrierter Steuerungssysteme erforderlich, welche neben quantitativen und vergangenheitsorientierten auch qualitative und zukunfts- orientierte Kennzahlen berücksichtigen, ohne dabei jedoch an Praktikabilität zu verlieren.

Ein diesen Anforderungen entsprechendes integriertes Kennzahlensystem ist die BSC, mittels der die auf die Unternchmensziele ausgerichtete Leistungserbringung anhand weniger ausgewählter Kennzahlen multikriteriell beurteilt werden kann. In Anbetracht der Rahmenbedingungen, die sich u. a. aus dem Programmauftrag, der gesetzlich kodifizierten Beachtung des Wirtschaftlichkeits- und des Sparsamkeitsprinzips, den heterogenen Selbstfinanzierungspotenzialen (Gebühren und Werbeeinnahmen) sowie der Organisationsstruktur öfentlich-rechtlicher Anbieter ergeben, wurde eine hierarchische Scorecard-Mappe vorgeschlagen, die anschließend mit der Top-BSC für die oberste Unternehnensebene konkretisiert wurde. Dabei wurden spezifische Perspektiven vorgestellt, welche aus einer Anpassung und Substitution der klassischen Betrachtungsebenen resultieren.

Vor allem beim Einsatz der BSC im öffentlich-rechtlichen Rundfunk ist mit vieleu Restriktionen und Behinderungen aus sämtlichen Feldern der Organisation zu rechnen. Die $\Lambda$ kzeptanz bei Mitarbeitern und Unternehmensführung wird somit zum wichtigsten Erfolgskriterium. Daher ist es von entscheidender Bedeutung, dass antreibende und akzeptierte Führungskräfte der obersten Unternehmensebene das BSC-Projekt aktiv unterstützen sowie dafür sorgen, dass das Konzept unternebmensintern professionell und als integrierter Ansatz kommuniziert wird. Dabei ist zu berücksichtigen, dass umso stärkere Promotoren benötigt werden, je innovativer die Anwendung ist bzw. je „,eingefahrener" einzelne Abteilungen und Direktionen sind. So ist bspw. davon auszugehen, dass in der Redaktionsabteilung, welche den Online-Auftritt verantwortet, mit weniger "Widerstand“ zu rechnen ist als in den jahrelang bestehenden Abteilungen der Verwaltungsdirektion. Offentlichrechtliche Rundfunkunternehmen sollten zudem berïcksichtigen, inwieweit sie vollständig eigenständig die benötigten quantitativen und qualitativen Ressourcen, die die Umsetzung eines solchen Projektes crfordert, zur Verfügung stellen können. Vielfach wurden positive Erfahrungen in der Zusammenarbeit und externen Beratern gemacht, welche ergebnisorientiert die Implementierung eines Balanced-Scorecard-Projektes vorantreiben können (vgl. hierzu Kaufmann 1997, S. 427 f.).

\section{Literatur}

ABPLANAI.P, P./LOMBRISER, R., Unternehmensstrategie als kreativer Prozeß, München 2000. BÖRNER, C. J., Strategisches Bankmanagement: ressourcen- und marktorientierte Strategien von Universalbanken, München et al. 2000.

BRÖSEl., G., Zur Daseinsberechtigung des öffentlich-rechtlichen Rundfunks, in: ZögU, 26. Jg., 2003, S. 115-131.

BORKI, D. M., Der ,resource-based view"-Ansat?. als neues Denkmodell des strategischen Managements, Diss., St. Gallen 1996.

Degenhiart, C., Funktionsauftrag und „dritte Programmsäule" des öffentlich-rechtlichen Rundfunks, in: Kommunikation \& Recht, 4. Jg. (2001), S. $329-338$

GLXSER, M., Schlüsselfaktoren für das erfolgreiche Controlling im öffentlichen Rundfunk, in: krp, 43. Jg. (1999), S. 301-309.

HEINRICH, J., Medienökonomie, Band 1, 2. Aufl., Wiesbaden 2001.

Horvarth \& Partnir (Hrsg.), Balanced Scorecard umsetzen, Stuttgart 2001.

Kaplan, R. S./Norton, D. P., Balanced Scorecard - Strategien erfolgreich umsetzen, Stuttgart 1997.

KaUf:MANN, L., Balanced Scorecard, in: ZP, 8. Jg. (1997), S. 421-428.

KFuPER, F, Strategisches Management, München 2001.

Keupre, F./HANS, R., Multimedia-Management, Wieshaden 2003.

KeuPER, F./HANS, R., Erlösformen in Geschäftsmodellen der Medienbranche, in: SCHOLZ, C. (Hrsg.), Handbuch zum Management in Medienunternehmen, Baden-Baden 2004 (im Druck).

KOCHER, A., Controlling der werbefinanzierten Medienunternehmung, Lohmar et al. 2002.

Prafinlad, C. K./HAMEl, G., The Gore Competence of the Corporation, in: Harvard Business Review, 1990, Nr. 3, S. 79-91.

SCheillhasss, H. M., Rundfunkökonomie, in: DBW, 60. Jg. (2000), S. 531-534.

SifrFi, G./SCHWERTZE!, U., Materialien zur Rundfunkökonomie II; Management für Rundfunkunternehmen - Teil I, Arbeitspapiere des Instituts für Rundfunkökonomie, H. 113, 2. Aufl., Köln 1997.

ZDF, ZDF über das ZDF: Der Funktionsanftrag, online: http://www.zdf.de/ZDFde/inhalt/ $30 / 0,18$ 72,2001950,00.html, Stand: unbekannt, Abruf: 11.12.2003 